EPJ Web of Conferences 59, 03011 (2013)

DOI: $10.1051 /$ epjconf/20135903011

(C) Owned by the authors, published by EDP Sciences, 2013

\title{
Progress and prospects for an IFE relevant FI point design
}

\author{
M. Key, P. Amendt, C. Bellei, D. Clark, B. Cohen, L. Divol, D. Ho, A. Kemp, \\ D. Larson, M. Marinak, P. Patel, H. Shay, D. Strozzi and M. Tabak
}

Lawrence Livermore National Laboratory, Livermore, CA 94550, USA

\begin{abstract}
The physics issues involved in scaling from sub-ignition to high gain fast ignition are discussed. Successful point designs must collimate the electrons and minimise the standoff distance to avoid multimegajoule ignition energies. Collimating B field configurations are identified and some initial designs are explored.
\end{abstract}

Fast ignition (FI) is an advanced concept for inertial confined fusion (ICF) that has the potential to deliver gain $\sim 100 \times$ for a driver energy $\sim 1$ MJ. It is attractive for the development of inertial fusion energy (IFE) because target performance in this regime would be most effective for an IFE power plant. It is currently less attractive than central hot spot (CHS) ignition because it lacks a credible point design for high gain. Here we discuss our work to develop such a point design.

Experimental research on FI and much of the numerical modeling effort have been focused on near term sub-ignition-scale FI experiments that are accessible with current laser facilities. The issues arising with scaling up to high gain have had less attention. High gain requires $\rho \mathrm{R} \sim 3 \mathrm{gcm}^{-2}$ whereas $\rho \mathrm{R}$ values in leading FI experiments (e.g., FIREX I and OMEGA EP cone guided electron FI studies) are an order of magnitude smaller.

Increase of $\rho \mathrm{R}$ is obtained by self similar scaling of the hydrodynamics of the target. This brings two difficulties which lead to reduced coupling efficiency of laser energy to the ignition hot spot. Distances scale in proportion to $\rho \mathrm{R}$ so that the stand off between the cone tip and the dense fuel is increased, reducing the coupling efficiency for a divergent electron source. Scaled up thickness of the cone tip increases the energy loss and scattering of the electrons which also reduces the coupling efficiency.

Measurements of the divergence of the electrons in initially cold solid targets include significant selfcollimation by azimuthal B fields and therefore underestimate the true source divergence. Large-scale particle in cell (PIC) modeling has indicated higher source divergence with a normalized integrated solid angle $\sim 5$ steradians [1]. While the results of the PIC modeling lack adequate validation by experimental benchmarking, they are currently the best available guides to the source characteristics.

We use the modeling capability and methodology from the indirect-drive CHS point design for the national ignition campaign (NIC), in particular the Lasnex and Hydra radiation-hydrodynamic codes. A 3D/2D PIC code, PSC [2] provides large-scale modeling of the laser plasma interaction and the electron source [1]. Electron transport is modeled with a hybrid PIC code, Zuma, and it has been integrated with Hydra to model ignition and burn [3]. An important corollary of our code development is our experimental studies aimed at benchmarking the PIC and hybrid PIC modeling.

Figure 1 illustrates a 2D hydro design of an ignition-scale target with an Au cone and diamond cone tip. It has $2.75 \mathrm{mg}$ of DT fuel and is driven with a $1.75-\mathrm{MJ}$ laser pulse at 0.35 micron wavelength. If ignited it gives $277 \mathrm{MJ}$ of fusion energy (gain 150x neglecting the ignitor energy). There is, however, 110 micron of standoff distance between the inner surface of the cone and the dense fuel. This stand off is already reduced relative to a symmetric implosion, by $50 \mu \mathrm{m}$ of $\mathrm{P} 1$ asymmetry in shell radius, which

This is an Open Access article distributed under the terms of the Creative Commons Attribution License 2.0, which permits unrestricted use, distribution, and reproduction in any medium, provided the original work is properly cited. 


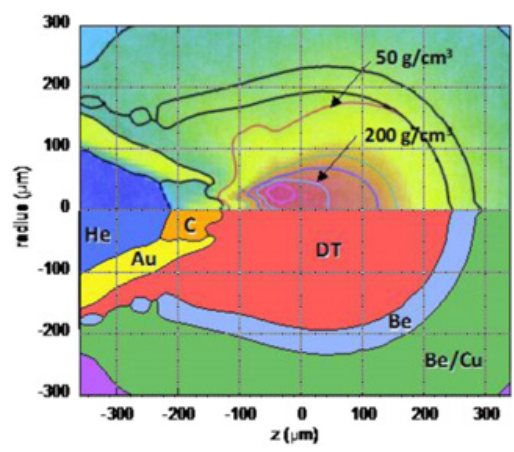

Figure 1. Cone coupled FI fuel assembly design for high gain.

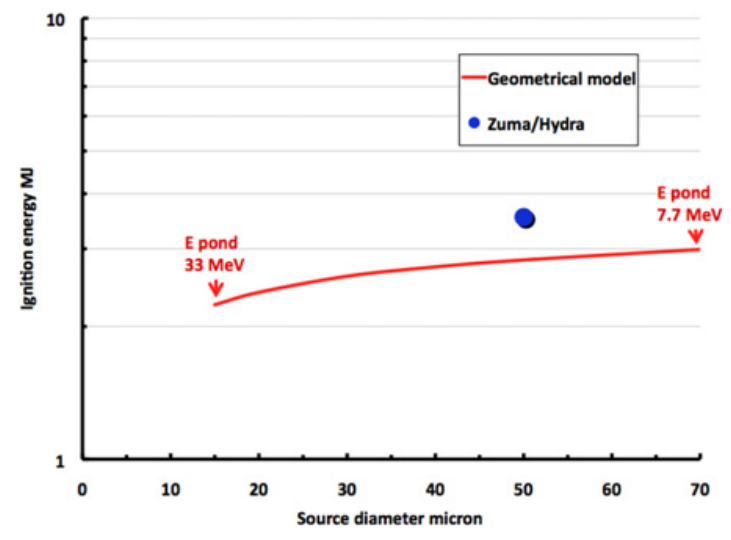

Figure 2. Ignition energy of DT at $450 \mathrm{~g} / \mathrm{cc}$ for a $0.53 \mu \mathrm{m}$ laser, $15 \mathrm{ps}$ pulse and $70 \mu \mathrm{m}$ standoff.

causes the implosion to arrive earlier at the center from the cone side. The peak fuel density reaches $300 \mathrm{~g} / \mathrm{cc}$, which is suitable for FI, but the standoff distance is large enough to present major problems for electron ignition.

We use analytic fits to PIC modeling results from PSC to define the divergence and the energy spectrum of the electron source injected in Zuma, and we scale the energy spectrum with the laser intensity in proportion to the ponderomotive potential based on PSC modeling at higher intensities [4]. The Zuma /Hydra coupled codes model ignition and burn. An idealized spherical DT fuel mass is assumed with $450 \mathrm{~g} / \mathrm{cc}$ peak density and $70 \mu \mathrm{m}$ radius. A solid C cone of density $20 \mathrm{~g} / \mathrm{cc}$ with a $50-\mu \mathrm{m}-$ diameter tip and a $60^{\circ}$ cone angle is placed $70 \mu \mathrm{m}$ from the fuel mass. An electron source is launched in a plane $20 \mu \mathrm{m}$ inside the cone tip. Ignition occurs at 3.5-MJ laser energy in a 15-ps pulse at 0.53 -micron laser wavelength for a $50 \mu \mathrm{m}$ source diameter.

This unacceptably large ignition energy is due to the large source divergence and stand off distance. The divergence suppresses any auto-induced collimation by azimuthal B fields. Geometrical calculation of the electron flux density on axis and its absorption efficiency in the $1.2 \mathrm{gcm}^{-2}$ of the ignition hot spot is used with published results on the lowest absorbed electron energy flux density needed for ignition (the width $>$ depth mode) [5], to calculate the ignition energy.

Figure 2 shows both the previously described Zuma Hydra calculation and the geometrical modeling results. The underlying scaling follows from using the normalized integrated solid angle $\Omega$ to define the beam intensity on axis $\mathrm{I}(0)=\mathrm{P} /\left(\Omega \mathrm{z}^{2}\right)$, (valid at distances significantly greater than the source diameter). The coupled fraction of the flux scales as the electron energy $\mathrm{E}^{-1}$ and $\mathrm{E}$ is proportional 
IFSA 2011
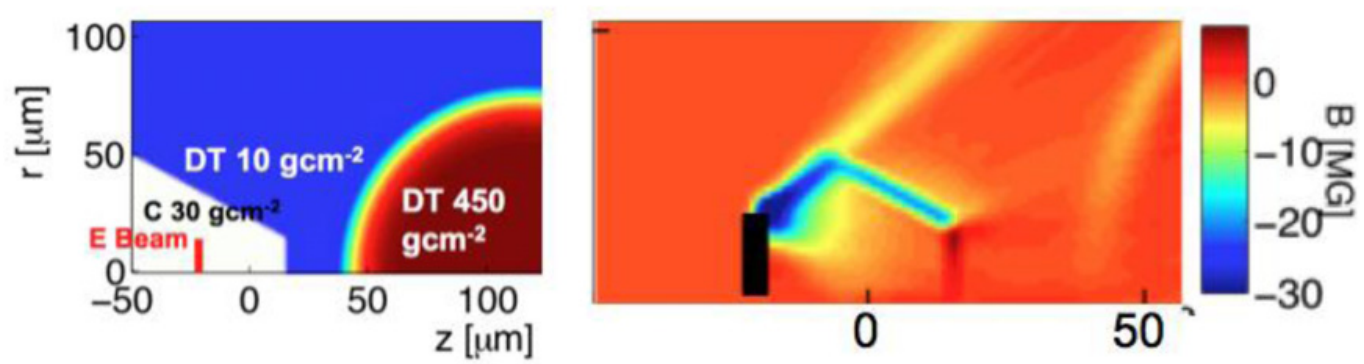

Figure 3. Left: initial conditions. Right: auto induced B field in the cone tip.

to the ponderomotive potential, which scales as $\left(\mathrm{I} \lambda^{2}\right)^{0.5}$. The ignition power therefore scales as $\left(\Omega \mathrm{z}^{2}\right)^{2}$, or as ( standoff $\times$ divergence). ${ }^{4}$

An effective way to collimate electrons is to use an axial magnetic field in which the electron Larmor radius is smaller than the ignition hot spot radius so that electrons spiral along the field lines from the laser focal spot to the ignition hot spot [6]. The required B field strength is $>50 \mathrm{MG}$, and ignition energies at $450 \mathrm{~g} / \mathrm{cc}$ are reduced to $\sim 260 \mathrm{~kJ}$ of 0.53 micron laser energy [7]. Preformed 50-kG fields can be compressed in the FI implosion to $>50 \mathrm{MG}$, but unfortunately B field diffusion is too slow for effective penetration inside the FI target cone tip. The consequence is that electrons must travel from a low axial B field to a high field and encounter mirror reflection. Efficient escape through the mirror requires a mirror loss cone angle as large as the source cone angle, and analytic estimates suggest this limits the B field increase to $<30 \%$. Numerical modeling results [7] confirm a strong increase in ignition energy for B field increases $>50 \%$. This avenue of electron collimation is therefore blocked by mirror reflection.

An alternative collimation method is use of a B field with hollow pipe geometry such that electrons are reflected and confined within the pipe, which guides them to the ignition hot spot. The reflection condition for angle $\theta$ and field thickness $\mathrm{L}$ is: $\mathrm{BL}>\left(\gamma_{\mathrm{e}} \mathrm{v}_{\mathrm{e}} \mathrm{m}_{\mathrm{e}} / \mathrm{e}\right)(1-\cos \theta)$. A converging configuration could force the beam into a smaller area allowing a lower intensity and cooler electron temperature at the source to improve coupling efficiency, but conservation of emittance implies that the beam solid angle at the exit would increase inversely with the beam area. In addition, for steep convergence angles, electrons are reflected back to the source after a few reflections. Converging configuations are therefore not helpful.

There are two possible routes to hollow fields. One is compression of an axial B field around a cylinder of dense material that resists compression [7]. The other is auto-generation of an azimuthal B field by the electron beam using a radial change of resistivity $\eta$ in a current density j where $\mathrm{dB} / \mathrm{dt} \sim \nabla \eta$ $\mathrm{x} \mathrm{j}$ [8]. We have explored the latter in more detail. Ohmic heating limits $\mathrm{B}$ field growth by reducing resistivity so that the achieved $B$ field scales as $B \sim \mathrm{j}^{-1}$ in a time $\mathrm{t} \sim \eta^{-1} \mathrm{j}^{-2}$. A Zuma /Hydra model calculation with a slightly different set up than the example in figure 2 is shown in Fig. 3. A 30-microndiameter source in an idealized cone/fuel configuration, achieves ignition at 1.6 MJ of e-beam with a 20 ps pulse of $0.53-\mu \mathrm{m}$ wavelenth. The cone tip is 35 micron from the fuel and the source is 35 micron inside the cone tip. The 15-MG B field at the edge of the cone is not an ideal collimator, being strong enough only to reflect $<3 \mathrm{MeV}$ électrons while the ponderomotive potential is $32 \mathrm{MeV}$.

Stronger B fields are generated with higher Z materials which have higher resistivity, and we have begun hydro design studies to explore how best to create cone tip protrusions that will generate stronger azimuthal B fields. For example a design with an Au protruding tip has only a $20-\mu \mathrm{m}$ stand off in DT, but the $\mathrm{Au}$ is compressed and has in addition to its high $\mathrm{Z}$, an axial $\rho \mathrm{L}$ of $4.4 \mathrm{gcm}^{-2}$. Scattering and absorption, therefore, prevent effective propagation of the electrons. Figure 4 shows a compromise 


\section{EPJ Web of Conferences}

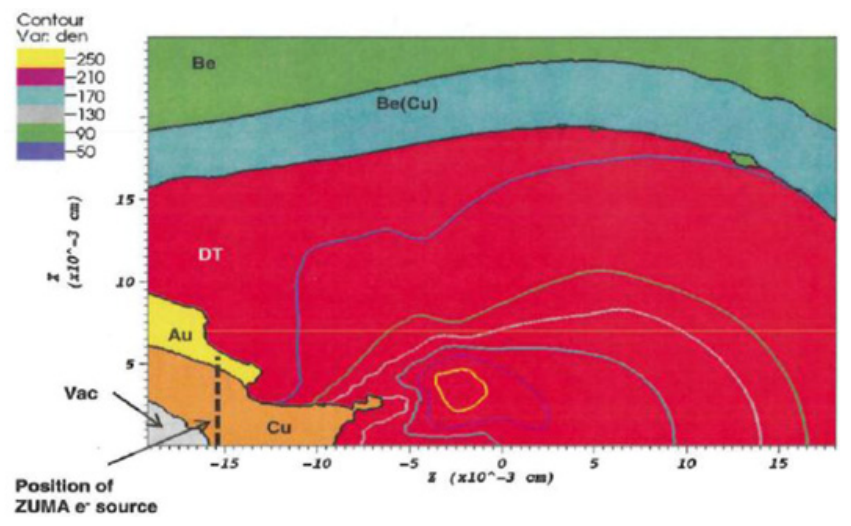

Figure 4. Hydra 2D target design with a protruding $\mathrm{Cu}$ tip showing the location of the electron source used for Hydra /Zuma modeling of electron transport and heating.

using $\mathrm{Cu}$ as the cone tip material. Here, the axial $\rho \mathrm{L}$ is $1.4 \mathrm{gcm}^{-2}$ and the stand off distance from the $\mathrm{Cu}$ to the dense DT is $50 \mu \mathrm{m}$.

This example has been used to test the full Hydra /Zuma capability by injecting electrons at the perpendicular source plane shown in Figure 4. The planar source is idealized since a source created by a laser would be located on the non-planar inner surface of the cone tip. Injection of up to $2.2 \mathrm{MJ}$ in $20 \mathrm{ps}$ with a source diameter of 50 micron and $0.53-\mu \mathrm{m}$ laser wavelength did not, however, produce ignition, indicating the inadequacy of this design. Use of still lower $\mathrm{z}$ materials in the cone tip that have lower density, leads to more push back by the hydro jet from the implosion core. We are exploring how to optimize this scheme.

We have not yet found a viable point design but we are at an early stage in our use of well-adapted design tools, and there is a huge design space that we have yet to explore. It is also important to ensure that the source divergence is correctly specified. The PIC modeling is still without adequate benchmarking, nor has it been fully explored. Recent PIC simulations with longer 5-ps pulse duration suggest reduced divergence for the higher energy electrons. Development of an adequate point design for fast ignition remains a crucial requirement. It is a large task and will require time and effort commensurate with its scale. Fast ignition has had only a tiny fraction of the design effort used to develop the CHS point design for the NIC.

This work was performed under the auspices of the U.S. Department of Energy by Lawrence Livermore National Security, LLC, Lawrence Livermore National Laboratory under Contract DE-AC52-07NA27344.

\section{References}

[1] A.J. Kemp, and L.Divol, submitted to Phys. Rev. Lett. (2011)

[2] B.I. Cohen, A. J. Kemp, and L. Divol, J. Comput. Phys. 229, 4591 (2010)

[3] M.M. Marinak, D. Larson, H. D. Shay, D. Ho, Bull. Am. Phys. Soc. 55, 182 (2010)

[4] D.J. Strozzi et al., in these proceedings

[5] S. Atzeni, A. Schiavi and C. Bellei, Phys. Plas. 052702, 14 (2010)

[6] M. Tabak et al., Bull. Am. Phys. Soc. DPP, JP9, 105 (2010)

[7] D.J. Strozzi et al., Phys. Plas. 19, 072711 (2012)

[8] A.P.L. Robinson, and M Sherlock, Phys. Plas. 14, 083105 (2007) 